\title{
A new smooth contact element: 3D diffuse contact element
}

\author{
D. Chamoret ${ }^{1, a}$, A. Rassineux ${ }^{2}$ and J.M. Bergheau ${ }^{3}$ \\ 1 Laboratoire M3M, UTBM, Site de Sévenans, 90010 Belfort Cedex, France \\ 2 Laboratoire Roberval, FRE 2833 CNRS/UTC, BP 20.529, 60205 Compiègne Cedex, France \\ 3 LTDS, UMR5513, CNRS/ECL/ENISE, 58 rue Jean Parot, 42023 Saint-Étienne, France
}

Received 27 August 2007, accepted 14 January 2008

\begin{abstract}
Many difficulties due to geometrical and material non-linearities arise when dealing with numerical simulation of contact problems. Within a finite element context, the contact interface is usually represented by a piecewise differentiable surface. Numerical problems due to the non-smoothness of the contact surface may occur especially when large slips are considered. Major changes of normal and tangential vectors may impede both convergence and precision. In order to smooth the contact interface and to release constraints due to the mesh, we propose a technique in which diffuse approximation is combined with a determination of neighboring nodes by a convex hull strategy. The formulation is developed for three-dimensional applications with frictionless contact and a $3 \mathrm{D}$ diffuse contact element has been developed. The efficiency of the approach has been validated with industrial frictionless contact problems.
\end{abstract}

Key words: Contact modelling; finite element method; implicit method; smooth contact surface; diffuse approximation; contact element; neighbourhood criterion; convex hulls

\section{Introduction}

The simulation of contact remains an important challenge for engineers. A variety of problems are concerned with contact phenomenom for instance metal forming processes (stamping, metal bottle cap as shown in Fig. 1), rubber sealings, crash analysis of cars, rolling contact between car tyres and the road. In this context, numerical instabilties may occur and impede the convergence process. Moreover contact problems can be associated either with large elastic or inelastic deformations. Several approaches have been proposed. Small deformation contact problems can be suitably modelled by node-to-node contact element. When dealing with large slips, nodeon-segment contact algorithms can be used [4]. In this context, powerful contact research algorithms have been presented [3, 11, 13, 17, 21, 24, 34, 35]. Most of these algorithms have a complex implementation and any lack of robusteness may impede the convergence of the process especially when the equilibrium equation are handled by implicit schemes. Numerical problems due to the non-smoothness of the contact surface may occur especially when large slips are considered. Major changes of normal and tangential vectors may impede both convergence and precision. Moreover, these effects may alter the residual vector and impede convergence. A number of authors have proposed techniques to smooth or average the normal vector $[14,26,30]$. The determination of a pseudo normal vector varying continuously in the neighbourhood

\footnotetext{
${ }^{a}$ Corresponding author: dominique.chamoret@utbm.fr
}
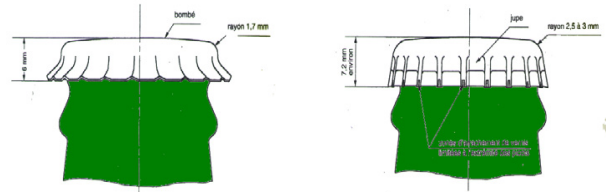

(a) Geometrical model
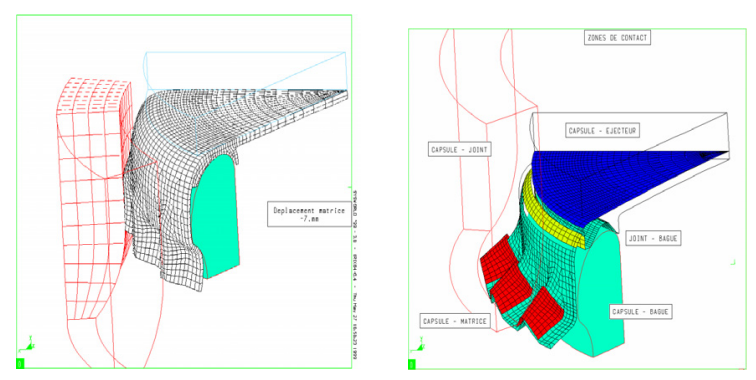

(b) Finite element model

Fig. 1. Metal bottle cap.

of contact node have been proposed in [22]. Interpolation techniques based on curved patches can be used [28]. Rigid contact surfaces are smoothed by techniques used 
in CAD surface modeling [15]. Bézier or B-Splines can be used with efficiency to represent deformable contact surfaces $[12,18,25,27,32]$.

The approach presented here consists in creating a smooth representation of the contact surface with the use of a meshfree technique denoted as diffuse approximation [23]. The interpolation of a geometrical model using a second order equation has been introduced by Rassineux in a remeshing context of discrete data [29]. We have extended this technique to the smoothing of the contact geometry [7]. Belytschko has proposed a similar approach [2]. The discretization of the problem leads to the creation of a 3D diffuse contact element. When dealing with meshfree techniques, nodal shape functions are not given by element connectivity but by a set of neighboring nodes. When dealing with the Finite Element Method, the influence of the orientation of the mesh on the solution is a well-known academic problem. In order to overcome this problem in a meshfree context, we propose a searching technique based on convex hulls determination [9]. The determination of all the contact elementary quantities is detailed and the efficiency of this original approach illustrated by examples.

The following outline is used in the presentation. Basic concepts of contact modelling are reviewed in part 2 . The contact surface smoothing procedure is presented in part 3. New contact geometry is illustrated in part 4 . The finite element method and our approach are combined to construct a new contact element proposed in part 5. Examples which demonstrate the efficiency of the proposed algorithms are discussed in part 6 .

\section{Contact modelling}

We provide a brief presentation of techniques frequently used to solve contact problems in a FEM context $[1,10,16,19,20,31]$.

\subsection{Contact geometry and gap function}

Let us consider two bodies in potential contact. In a master-slave approach, the slave body is denoted as $\Omega^{s}$ and the master body is denoted as $\Omega^{m}$. The same terminology is used for surfaces. The potential contact surfaces are noted $\gamma_{c}^{i}$ with $i=s, m$ in the current configuration. The master body is assumed to be the reference body which means that the relative motion of the slave body is described with respect to the master body. The condition to ensure is that the slave body must not penetrate the master body and all displacements are computed with respect to this assumption. This situation introduces dissymmetry in the description of the problem. However this drawback can be reduced numerically by alternating the role of the bodies with the help of specific algorithms [4].

The aim is to measure the penetration by computing the minimal distance between the two surfaces into contact. At each slave point $\boldsymbol{x}^{c}$ of the surface $\gamma_{c}^{s}$ is associated

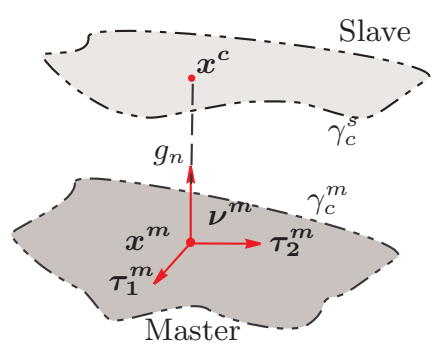

Fig. 2. Representation of the normal gap.

a point $\boldsymbol{x}^{\boldsymbol{m}}$ belonging to the master surface $\gamma_{c}^{m}$ given by the following minimization problem:

$$
\left\|\boldsymbol{x}^{c}-\boldsymbol{x}^{\boldsymbol{m}}\right\|=\min _{x \in \gamma_{c}^{m}}\left\|\boldsymbol{x}^{c}-\boldsymbol{x}\right\| .
$$

This point is the closest projection point of $\boldsymbol{x}^{\boldsymbol{c}}$ onto the master surface. Consequently, vector $\boldsymbol{x}^{c}-\boldsymbol{x}^{\boldsymbol{m}}$ and normal vector $\boldsymbol{\nu}^{m}$, have the same direction and we can establish the relation:

$$
x^{c}-x^{m}=g_{n} \nu^{m}
$$

$b^{m}=\left(\boldsymbol{\tau}_{\mathbf{1}}^{\boldsymbol{m}}, \boldsymbol{\tau}_{\mathbf{2}}^{\boldsymbol{m}}, \boldsymbol{\nu}^{\boldsymbol{m}}\right)$ is the natural basis associated with the master surface in the current configuration at point $\boldsymbol{x}^{\boldsymbol{m}}$ (see Fig. 2). The normal gap function, denoted as $g_{n}$ is given by:

$$
g_{n}=\left(\boldsymbol{x}^{c}-\boldsymbol{x}^{\boldsymbol{m}}\right) \cdot \boldsymbol{\nu}^{\boldsymbol{m}} .
$$

The normal gap has a major importance in the description of the penetration between the two bodies. The sign of the gap provides the geometrical status of a contact point. Three situations may occur:

$$
\left\{\begin{array}{cc}
\text { No contact } & g_{n}>0 \\
\text { Perfect contact } & g_{n}=0 \\
\text { Interpenetration } & g_{n}<0
\end{array}\right.
$$

\subsection{Numerical schemes to solve contact problems}

The numerical treatment of the contact constraints is essentialy based on two main strategies: penalty and Lagragian multiplier based methods. Both approaches have their advantages and their drawbacks. A penalty method can be easily implemented in an existing finite element code. This is the reason why it is used so frequently. The penalty method estimates the normal contact traction as:

$$
T_{n}=\left\{\begin{array}{l}
-\varepsilon_{n} g_{n} \text { if } g_{n} \leq 0 \\
0 \quad \text { otherwise }
\end{array}\right.
$$

where $\varepsilon_{n}$ is the normal penalty parameter and $g_{n}$ the normal gap.

The main disadvantage of this technique is the adequate choice of the penalty parameter and the convergence of the technique is highly dependent on this choice. An unwise choice may lead to ill-conditioned stiffness matrix if the penalty parameter is too important or to unacceptable penetration if it is too small. Solutions to adjust the penalty parameter have been proposed [8]. 


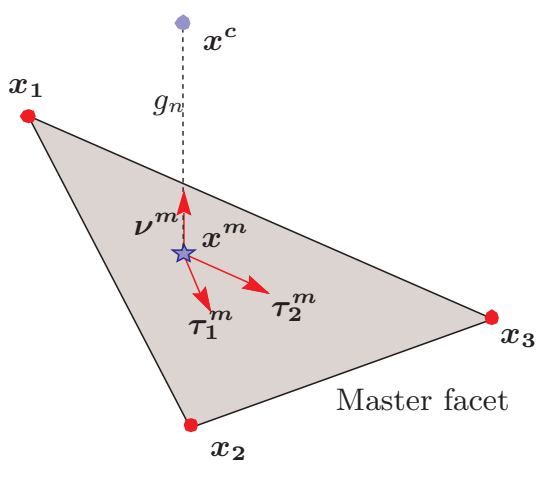

Fig. 3. Node-to-facet contact element.

\subsection{Weak form}

The variational problem is classicaly written as

$$
G(\boldsymbol{u}, \boldsymbol{\delta} \boldsymbol{u})+G_{c}(\boldsymbol{u}, \boldsymbol{\delta} \boldsymbol{u})=0
$$

where $\boldsymbol{u}$ is the solution displacement field. Function $\boldsymbol{\delta} \boldsymbol{u}$ can be seen as virtual displacements. First term $G$ is the virtual work associated with finite deformation of solids mechanics problems.

Second functional $G_{c}$ contains the contact contributions. These contributions $[19,31]$ can be expressed as follows:

$$
G_{c}(\boldsymbol{u}, \boldsymbol{\delta} \boldsymbol{u})=\int_{\gamma_{c}^{s}} \varepsilon_{n} g_{n} \delta g_{n} d \gamma
$$

where $\delta g_{n}$ is the first derivative of $g_{n}$.

The problem introduced by relation (5) is nonlinear and can be solved by a Newton Raphson procedure which requires the computation of derivatives. Using a continuous description of the contact, the linearization of contact contributions can be written as:

$$
\Delta G_{c}(\boldsymbol{u}, \boldsymbol{\delta} \boldsymbol{u})=\int_{\gamma_{c}^{s}}\left(\varepsilon_{n} \Delta g_{n} \delta g_{n}+\varepsilon_{n} g_{n} \Delta \delta g_{n}\right) d \gamma
$$

where $\Delta \delta g_{n}$ is the second derivative of $g_{n}$. We remark the crucial role of the gap and its variations in the contact contributions.

\subsection{Discretization: contact element}

When the finite element method is used, contact surfaces are discrete surfaces on which relation (6) must be discretized. Only one slave node is supposed to be in contact with the discretized master surface. Due to the variety of contact situations, specific contact elements have been developed such as node-to-node contact element for small deformations and node-to-face contact element for large deformations [33]. This element is composed of a slave node and nodes representing the master contact face (Fig. 3). The difficulty consists in expressing $g_{n}$ and its variations.

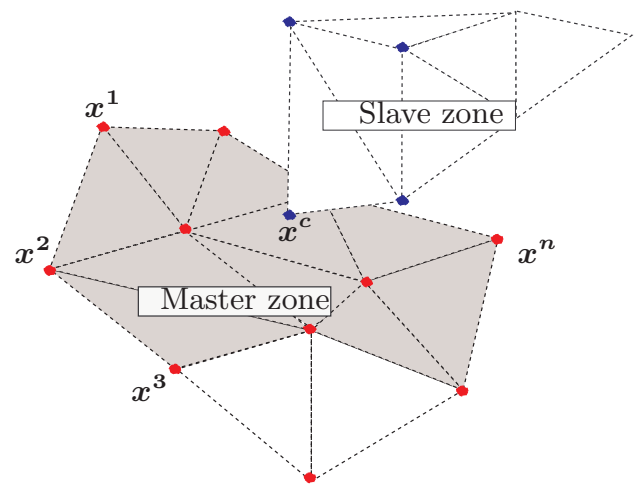

Fig. 4. Contact zones.

\section{Diffuse approximation and contact}

In this part, the building of the smooth contact surface is developed. Let us consider two potential contact zones denoted as master and slave zone. The master zone is made of a set of faces (such as triangles or quadrangles in a 3D context) from the finite element mesh of the master solid. Let $\boldsymbol{x}^{\boldsymbol{i}}$ denotes the position of a node $i$ from this set. The slave zone is made of a set of nodes likely to come into contact with the master zone (Fig. 4). The aim is to determine an approximation $S_{g}^{d}$ of the contact surface with the only data of nodes $\boldsymbol{x}^{\boldsymbol{i}}$.

A moving least square approach denoted as diffuse approximation technique has been used. The diffuse surface is built from a succession of local approximations. However, the coefficients of the surface depend on the evaluation point. The method guarantees that the coefficients vary in a continuous way with respect to the location of the point. The diffuse interpolation is given by the minimization of a criterion based on the interpolation of the set of neighboring nodes and can be written $S_{g}^{d}$. A local approximation of the contact surface $S^{d}$ and therefore, a set of neighboring nodes is associated to each slave node $x^{c}$

\subsection{Local approximation}

We suppose that we have determined a set $S_{i}$ of nodes $\boldsymbol{x}^{\boldsymbol{i}}$ belonging to the master surface in the neighborhood of $\boldsymbol{x}^{c}$, the evaluation point. We remark that the master surface is used to create the diffuse interpolated surface. The computation of an interpolated surface requires a set a $2 \mathrm{D}$ parametric coordinate system of the surface.

We suppose that at each point of the discrete surface, we can find a neighborhood and therefore a local coordinate system on which the surface can be locally defined by a Monge patch

$$
\mathrm{f}\left(X_{1}, X_{2}\right)=X_{3}
$$

where $\mathrm{f}$ is a $\mathrm{C}^{2}$ function defined on a planar domain.

Therefore, the first step consists in the determination of a moving least square plane (average plane) with the 
use of the interpolation set $\mathrm{S}_{\mathrm{i}}$. The surface equation is evaluated through a second order equation and can be expressed in the local coordinate system as

$$
\mathrm{f}\left(X_{1}, X_{2}\right)=\left\langle 1, X_{1}, X_{2}, X_{1}^{2}, X_{1} X_{2}, X_{2}^{2}\right\rangle \boldsymbol{\alpha}=\mathbf{p}^{t} \boldsymbol{\alpha} .
$$

In a local set of coordinates $\mathrm{R}^{\mathrm{d}}$ linked to all nodes $\boldsymbol{x}^{\boldsymbol{i}}$ and in the neighbourhood of $\boldsymbol{x}^{\boldsymbol{c}}$, the local approximation associated with node $\boldsymbol{x}^{c}$ is defined by a Monge patch:

$$
\mathrm{f}_{\boldsymbol{x}^{c}}\left(X_{1}, X_{2}\right)=X_{3}
$$

where $\mathrm{f}_{x^{c}}$ is a $\mathrm{C}^{2}$ function defined on a planar domain and $\left(X_{1}, X_{2}, X_{3}\right)$ are the coordinates of a point in $\mathrm{R}^{\mathrm{d}} \cdot \mathrm{f}_{\boldsymbol{x}^{c}}$ can be expressed in the local coordinate system as

$$
\mathrm{f}_{\boldsymbol{x}^{c}}\left(X_{1}, X_{2}\right)=\mathbf{p}^{t}\left(\boldsymbol{x}-\boldsymbol{x}^{c}\right) \boldsymbol{\alpha}
$$

where $\left(X_{1}^{c}, X_{2}^{c}, X_{3}^{c}\right)$ are the coordinates of $\boldsymbol{x}^{c}$ in $\mathrm{R}^{\mathrm{d}}$.

For each node $\boldsymbol{x}^{c}$, the neighbourhood is composed of all nodes $\boldsymbol{x}^{\boldsymbol{i}}$ with $i=1, \ldots, n$ belonging to the opposite master zone. Coordinates of a master node $\boldsymbol{x}^{i}$ in this reference frame are written in block letters $X_{k}^{i}$ with $k=1, \ldots, 3$. In order to define the approximation, the number of nodes $\boldsymbol{x}^{\boldsymbol{i}}$ must be at least equal to the size of the polynomial basis, i.e. 6 . The search of neighboring nodes is extended until the number of nodes is sufficient. Whenever the master contact zone does not contain enough nodes, a linear polynomial basis is used.

\subsection{Determination of the coefficient of the diffuse surface}

All quantities thereafter are computed in the local coordinates system $\mathrm{R}^{\mathrm{d}}$. The 6 coefficients are calculated by a moving least squares method, based on the minimisation of the difference between the altitude $X_{3}^{i}$ of a master node $\boldsymbol{x}^{i}$ and function $\mathrm{f}_{\boldsymbol{x}^{c}}$ evaluated at this node, which leads to the following criterion:

$$
\mathrm{J}_{\boldsymbol{x}^{c}}(\boldsymbol{\alpha})=\sum_{i=1}^{i=n} w\left(\boldsymbol{x}^{i}, \boldsymbol{x}^{\boldsymbol{c}}\right)\left(\mathbf{p}^{t}\left(\boldsymbol{x}^{i}-\boldsymbol{x}^{\boldsymbol{c}}\right) \boldsymbol{\alpha}-X_{3}^{i}\right)^{2}
$$

where $w\left(\boldsymbol{x}^{i}, \boldsymbol{x}^{\boldsymbol{c}}\right)$ for $i=1, \ldots, n$ are the weighting function associated with node $\boldsymbol{x}^{i}$.

Vector $\boldsymbol{\alpha}$ is given by the minimization of criterion $\mathrm{J}_{\boldsymbol{x}^{c}}$ and must be the solution of the system defined by:

$$
\mathrm{P}^{t} \mathrm{~W} \mathrm{P} \boldsymbol{\alpha}=\mathrm{P}^{t} \mathrm{~W} \mathbf{Z}
$$

$\mathbf{Z}$ is the vector of the altitudes of all nodes $\boldsymbol{x}^{\boldsymbol{i}}$ and $\mathrm{P}$ is composed of polynomial basis $\mathbf{p}$ evaluated at each node $\boldsymbol{x}^{\boldsymbol{i}}$. W is the diagonal matrix of the weights.

The resolution of $6 \times 6$ system (13) leads to the determination of $\boldsymbol{\alpha}$ and thus to the knowledge of the local interpolation of the contact surface associated with node $\boldsymbol{x}^{c}$.

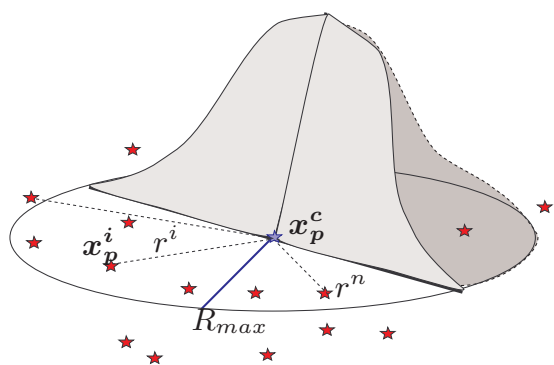

Fig. 5. Domain of influence.

\subsection{Weighting functions}

The computation of the weighting function has a major influence on the determination of the diffuse surface.

\subsubsection{Domain of influence}

For each point $\boldsymbol{x}^{c}$, only the nearest nodes $\boldsymbol{x}^{i}$ are taken into account. The contribution of each nodal value to the approximation is influenced by a weighting function $w^{i}$ such that $w^{i}>0$ inside the domain of influence of node $i$ and $w^{i}=0$ otherwise, providing a local character to the approximation. Fundamental properties related to MLS approximation, such as locality and continuity mainly depend on an appropriate choice of the weighting functions. In order to limit the number of nodes used for the local evaluation, the support of the approximation must be bounded. The issues relative to the construction and to the choice of different weighting functions are detailed in references $[5,6]$. In our context, the existence of the approximation requires a number of nodes at least equal to 6 at each evaluation point. Weighting function are radial function, the influence of which decrease with the distance to the node as shown in Figure 5. The domain of influence is centered at $\boldsymbol{x}_{\boldsymbol{p}}^{c}$ which is the projection of $\boldsymbol{x}^{\boldsymbol{c}}$ on the diffuse plane, and we assume that in the projection plane, the set of nodes is fully contained in a circle of radius $R_{\max } \cdot r^{i}$ denotes the distance between $\boldsymbol{x}_{\boldsymbol{p}}^{\boldsymbol{c}}$ and $x_{p}^{i}$, the projection of node $x^{i}$ on the diffuse plane. This distance is calculated in the projection plane.

\subsubsection{Value of the weight}

In a practical way, we have chosen a cubic polynomial function which is set to zero outside the domain of influence. The value of the weight is usually given by

$$
w^{i}=\left\{\begin{array}{ll}
(1-r)^{2}(1+2 r) & \text { if } 0 \leq r<1 \\
0 & \text { if } r \geq 1
\end{array} \text { where } r=\frac{r^{i}}{R_{\max }}\right.
$$




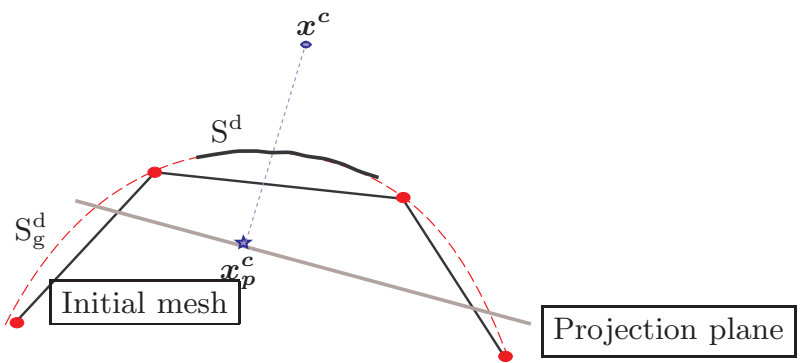

$\mathrm{S}_{\mathrm{g}}^{\mathrm{d}}$ : diffuse surface

$\mathrm{S}^{\mathrm{d}}$ : local approximation at note $\boldsymbol{x}^{c}$

Fig. 6. Local or global approximation?

\subsubsection{Diffuse approximation or diffuse interpolation?}

Diffuse approximation in its standard form does not interpolate data. The interpolation property is commonly obtained with weighting functions which has infinite value at the node:

$$
\boldsymbol{x}^{c} \rightarrow \boldsymbol{x}^{i} \Rightarrow w^{i} \rightarrow \infty .
$$

These functions are defined using the following substitution (Shepard):

$$
w^{i} \rightarrow \frac{w^{i}}{1-w^{i}}
$$

\section{New contact geometry}

A smooth contact surface has been built. We argued that the gap controls the whole contact process. We propose therefore to compute the gap using the intrinsic properties of the diffuse surface.

\subsection{Notations}

$\mathrm{f}_{\boldsymbol{x}^{\mathrm{c}}}$ is dependent on local coordinates $X_{1}^{c}, X_{2}^{c}$ and $X_{3}^{c}$, and on the coordinates of set of nodes $\boldsymbol{x}^{i}$, therefore, we use the following notations:

$$
\begin{gathered}
\boldsymbol{\alpha}=\boldsymbol{\alpha}\left(X_{1}^{c}, X_{2}^{c}, X_{3}^{c}, X_{1}^{1}, X_{2}^{1}, \ldots, X_{2}^{n}, X_{3}^{n}\right) \\
\mathrm{f}_{\boldsymbol{x}^{c}}\left(X_{1}, X_{2}\right)=\mathrm{f}\left(X_{1}, X_{2}, X_{1}^{c}, X_{2}^{c}, \ldots, X_{2}^{n}, X_{3}^{n}\right) .
\end{gathered}
$$

\subsection{Notion of diffuse gap}

The gap is defined as the minimal distance between the slave node $\boldsymbol{x}^{c}$ and the master surface. It is necessary to associate any slave node with a point belonging to the diffuse surface. This point is the projection which minimize the distance between $\boldsymbol{x}^{c}$ and the diffuse surface $\mathrm{S}_{\mathrm{g}}^{\mathrm{d}}$. The equation of the diffuse surface is not explicit and a succession of local approximations is necessary to define

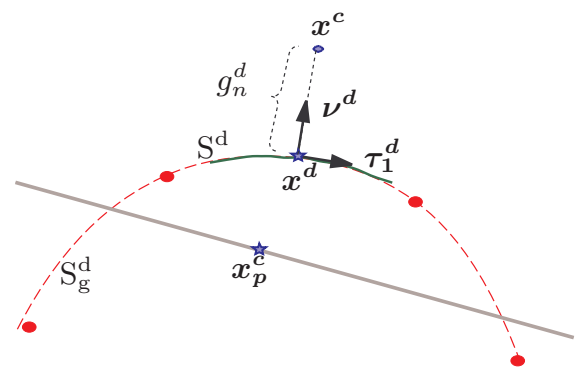

$\boldsymbol{x}^{d}: \quad$ projection of $\boldsymbol{x}^{c}$ on $\mathrm{S}^{\mathrm{d}}$

$g_{n}^{d}: \quad$ diffuse gap

$\nu^{d}$ : normal vector at the point $\boldsymbol{x}^{\boldsymbol{d}}$

$\tau_{1}^{d}$ : tangent vector at the point $x^{d}$

Fig. 7. Diffuse gap (2D representation).

it (Fig. 6). The idea is to use the local approximation at node $\boldsymbol{x}^{c}$ as the approximation of the surface.

A new point $\boldsymbol{x}^{\boldsymbol{d}}$ defined as the projection of $\boldsymbol{x}^{\boldsymbol{c}}$ on $\mathrm{S}^{\mathrm{d}}$ is introduced. A statement is made that the vector between $\boldsymbol{x}^{\boldsymbol{c}}$ and $\boldsymbol{x}^{\boldsymbol{d}}$ and the normal vector $\boldsymbol{\nu}^{\boldsymbol{d}}$ (associated with the surface $S^{d}$ at the point $\boldsymbol{x}^{\boldsymbol{d}}$ ) are collinear what leads to:

$$
\boldsymbol{x}^{c}-\boldsymbol{x}^{d}=g_{n}^{d} \boldsymbol{\nu}^{d} .
$$

Scalar $g_{n}^{d}$ (diffuse gap) describes the contact state on the regularized surface (Fig. 7).

\subsection{Local basis in $x^{d}$}

The Newton method can be used to determine $\boldsymbol{x}^{\boldsymbol{d}}$. Its coordinates are noted $\left(\bar{X}_{1}, \bar{X}_{2}, \bar{f}\right)$ where

$$
\overline{\mathrm{f}}=\mathrm{f}(\mathbb{X})
$$

with $\mathbb{X}=\left(\bar{X}_{1}, \bar{X}_{2}, X_{1}^{c}, \ldots, X_{i}^{j}, \ldots, X_{3}^{n}\right)$.

The tangent vectors at the point $\boldsymbol{x}^{\boldsymbol{d}}$ are given by:

$$
\boldsymbol{\tau}_{\mathbf{1}}^{\boldsymbol{d}}=\left[\begin{array}{c}
1 \\
0 \\
\frac{\partial \mathrm{f}}{\partial X_{1}}(\mathbb{X})
\end{array}\right] \text { and } \boldsymbol{\tau}_{\mathbf{2}}^{\boldsymbol{d}}=\left[\begin{array}{c}
0 \\
1 \\
\frac{\partial \mathrm{f}}{\partial X_{2}}(\mathbb{X})
\end{array}\right]
$$

The normal vector $\boldsymbol{\nu}^{\boldsymbol{d}}$ is the inner product of these two vectors:

$$
\nu^{\boldsymbol{d}}=\frac{\boldsymbol{\tau}_{\mathbf{1}}^{\boldsymbol{d}} \wedge \boldsymbol{\tau}_{\mathbf{2}}^{\boldsymbol{d}}}{\left\|\boldsymbol{\tau}_{\mathbf{1}}^{d} \wedge \boldsymbol{\tau}_{\mathbf{2}}^{\boldsymbol{d}}\right\|}=\frac{1}{\left\|\tau_{\mathbf{1}}^{d} \wedge \boldsymbol{\tau}_{\mathbf{2}}^{\boldsymbol{d}}\right\|}\left[\begin{array}{c}
-\frac{\partial \mathrm{f}}{\partial X_{1}}(\mathbb{X}) \\
-\frac{\partial \mathrm{f}}{\partial X_{2}}(\mathbb{X}) \\
1
\end{array}\right]
$$

\section{3D diffuse contact element}

We propose to combined the regularization of the contact surface with a specific search of neighboring nodes based 


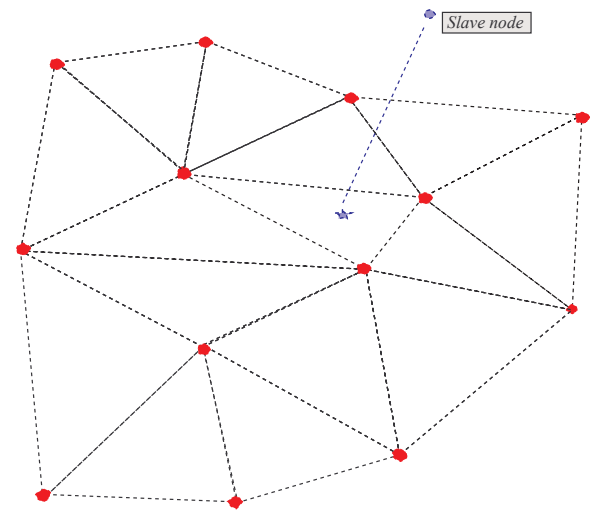

Fig. 8. $3 D$ diffuse contact element.

on convex hull strategy in order to create a 3D diffuse contact element.

The main difference between a traditional node-facet approach and our technique is that the contact is driven by potential contact nodes and not only by the nodes of the potential contact facet. Indeed, the slave and master surfaces can be seen as a set of nodes: a slave node $\boldsymbol{x}^{c}$ is supposed in contact with a master zone composed of $n$ nodes $\boldsymbol{x}^{i}$. The contact element we want to build is composed of a slave node $\boldsymbol{x}^{c}$ and $n$ neighbor nodes $\boldsymbol{x}^{\boldsymbol{i}}$ (Fig. 8). A neighbourhood criterion based on convex hulls determination is used to select nodes $\boldsymbol{x}^{i}$ used to build the local approximation.

\subsection{Neighbourhood criterion: convex hulls determination}

Contact areas are unknown a priori and may change considerably with the load step especially when large deformations occurs. The use of convex hulls in order to improve the quality of the approximation must be justified. The ideas is to capture the same number of nodes in all directions what cannot be obtained with a search based on a Euclidian metric. The computation of the distances is performed on the plane on which the interpolation surface has been determined. In order to optimize this step, we propose a searching technique based on convex hulls determination. In a first step, we solve the difficulties due to the anisotropy of the data points. When dealing with the Finite Element Method, the influence of the orientation of the mesh on the solution is a well-known academic problem. If a mesh-based approach is used to determine the neighborhood, the procedure can be carried out with the help of edges sharing the same node. However, we can remark that even in this context, the neighborhood depends on the swapping of the edges. Figure 9(a) shows that a search based on the nearest nodes of the mesh leads to the determination of 6 neighbors, when 8 (Fig. 9(b)) could be expected. In order to give to our work much emphasis, we decided to extend our techniques to set of points. Figure 10 illustrates the main steps of the technique. In a first step, $m$ points are selected with respect

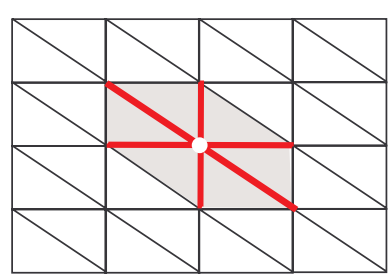

(a)

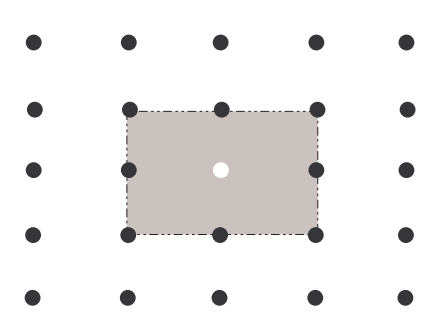

(b)

Fig. 9. Anisotropic neighborhood determination.

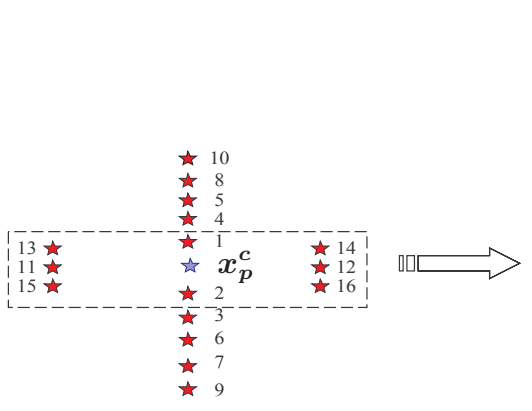

(a)

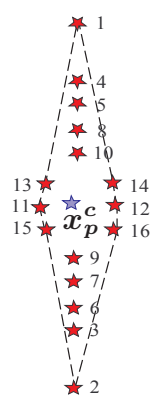

(b)
Fig. 10. Neighborhood determination by convex hull.

to their Euclidian distance at the evaluation point. Points are sorted and numbered with respect to their distance to the evaluation point. Each point is repositioned by inverting its distance to the origin. Then, the convex hull (using Graham scan) is determined in the inverted space as shown in Figure 10(a). The result of this procedure, a set of points in side a rectangular area, is displayed in Figure 10(b).

\subsection{Notations}

If $\boldsymbol{x}$ denotes a vector composed of the position of all the nodes, the first and the second variation of this vector are 
written:

$$
\boldsymbol{x}=\left[\begin{array}{l}
\boldsymbol{x}^{c} \\
\boldsymbol{x}^{1} \\
\boldsymbol{x}^{2} \\
\cdots \\
\cdots \\
\boldsymbol{x}^{n}
\end{array}\right] \quad \delta \boldsymbol{x}=\left[\begin{array}{l}
\delta \boldsymbol{x}^{c} \\
\delta \boldsymbol{x}^{1} \\
\delta \boldsymbol{x}^{2} \\
\cdots \\
\cdots \\
\delta \boldsymbol{x}^{n}
\end{array}\right] \quad \text { and } \quad \Delta \boldsymbol{x}=\left[\begin{array}{l}
\Delta \boldsymbol{x}^{c} \\
\Delta \boldsymbol{x}^{1} \\
\Delta \boldsymbol{x}^{2} \\
\cdots \\
\cdots \\
\Delta \boldsymbol{x}^{n}
\end{array}\right]
$$

In order to describe a 3D contact element, elementary residual vector and the tangent matrix must be determined using the features of the diffuse surface.

\subsection{Contact elementary residual vector}

In Section 2.3, we have shown that the first variation of $g_{n}^{d}$ must be computed to determine the contact residual vector.

\subsubsection{First variation of $g_{n}^{d}$}

Using relation (19), we can write

$$
\begin{aligned}
\delta g_{n}^{d} & =\delta\left\{\left(\boldsymbol{x}^{c}-\boldsymbol{x}^{\boldsymbol{d}}\right) \cdot \boldsymbol{\nu}^{d}\right\} \\
& =\delta\left(\boldsymbol{x}^{c}-\boldsymbol{x}^{\boldsymbol{d}}\right) \cdot \boldsymbol{\nu}^{\boldsymbol{d}}+g_{n}^{d} \boldsymbol{\nu}^{\boldsymbol{d}} \cdot \delta \boldsymbol{\nu}^{\boldsymbol{d}} .
\end{aligned}
$$

However $\left\|\boldsymbol{\nu}^{\boldsymbol{d}}\right\|=1$ which leads to:

$$
\nu^{d} \cdot \delta \nu^{d}=0
$$

Finaly, $\delta g_{n}^{d}$ can ne expressed as

$$
\delta g_{n}^{d}=\delta \boldsymbol{x}^{c} \cdot \boldsymbol{\nu}^{\boldsymbol{d}}-\delta \boldsymbol{x}^{\boldsymbol{d}} \cdot \boldsymbol{\nu}^{\boldsymbol{d}}
$$

In order to calculate $\delta \boldsymbol{x}^{d}$, the variation of $\overline{\mathrm{f}}$ must be determined. Using (17), it can be proved that

$$
\delta \overline{\mathrm{f}}=\frac{\partial \mathrm{f}}{\partial X_{1}}(\mathbb{X}) \delta \bar{X}_{1}+\frac{\partial \mathrm{f}}{\partial X_{2}}(\mathbb{X}) \delta \bar{X}_{2}+\delta \boldsymbol{x}^{t} \underset{\boldsymbol{x}}{\nabla \overline{\mathrm{f}}}
$$

where

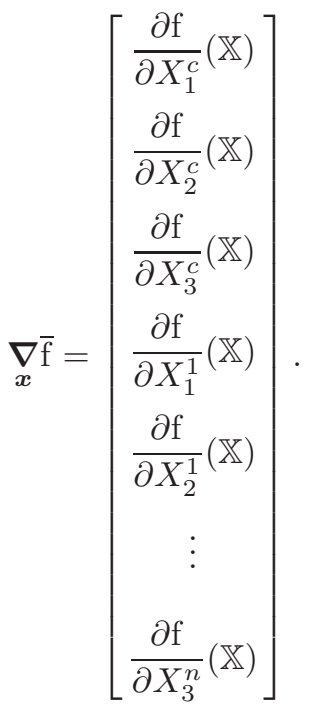

Using the definition of tangent vector and relation (27), we have

$$
\begin{gathered}
\delta \boldsymbol{x}^{\boldsymbol{d}}=\delta \bar{X}_{1} \boldsymbol{\tau}_{\mathbf{1}}^{\boldsymbol{d}}+\delta \bar{X}_{2} \boldsymbol{\tau}_{\mathbf{2}}^{\boldsymbol{d}}+\mathrm{d} \overline{\mathbf{f}} \\
\text { with } \mathrm{d} \overline{\mathbf{f}}=\left[\begin{array}{c}
0 \\
0 \\
\delta \boldsymbol{x}^{t} \underset{\boldsymbol{x}}{\boldsymbol{\nabla} \overline{\mathrm{f}}}
\end{array}\right]
\end{gathered}
$$

The expressions $\frac{\partial \mathrm{f}}{\partial X_{k}^{c}}(\mathbb{X})$ and $\frac{\partial \mathrm{f}}{\partial X_{k}^{i}}(\mathbb{X})$ for $k=1,2,3$ and $i=1, \ldots, n$ are obtained with the help of relation $(9)$ :

$$
\begin{aligned}
\frac{\partial \mathrm{f}}{\partial X_{k}^{c}}(\mathbb{X}) & =\frac{\partial \overline{\mathbf{p}}}{\partial X_{k}^{c}}{ }^{t} \boldsymbol{\alpha}+\overline{\mathbf{p}}^{t} \frac{\partial \boldsymbol{\alpha}}{\partial X_{k}^{c}} \text { for } k=1,2 \\
\frac{\partial \mathrm{f}}{\partial X_{3}^{c}}(\mathbb{X}) & =0 \\
\frac{\partial \mathrm{f}}{\partial X_{k}^{i}}(\mathbb{X}) & =\overline{\mathbf{p}}^{t} \frac{\partial \boldsymbol{\alpha}}{\partial X_{k}^{i}}
\end{aligned}
$$

where $\overline{\mathbf{p}}$ is the basis $\mathbf{p}$ evaluated at $\bar{X}_{1}$ and $\bar{X}_{2}$. The above expression show that $\frac{\partial \boldsymbol{\alpha}}{\partial X_{k}^{c}}$ and $\frac{\partial \boldsymbol{\alpha}}{\partial X_{k}^{i}}$ must be calculated.

\subsubsection{Discrete expression}

Introducing the value of $\delta \boldsymbol{x}^{d}$ in the definition of $g_{n}^{d}$, we obtain a discretized expression of the first variation of $g_{n}^{d}$ :

$$
\delta g_{n}^{d}=\delta \boldsymbol{x}^{t} \mathbf{N}_{\boldsymbol{c}}^{\boldsymbol{d}}
$$

where $\mathbf{N}_{\boldsymbol{c}}^{\boldsymbol{d}}$ is a $3(n+1)$ component vector,

$$
\mathbf{N}_{\boldsymbol{c}}^{\boldsymbol{d}}=\frac{-1}{\left\|\boldsymbol{\tau}_{\mathbf{1}}^{\boldsymbol{d}} \times \boldsymbol{\tau}_{\mathbf{2}}^{\boldsymbol{d}}\right\|}\left[\begin{array}{c}
\frac{\partial \mathrm{f}}{\partial X_{1}^{c}}(\mathbb{X})+\frac{\partial \mathrm{f}}{\partial X_{1}}(\mathbb{X}) \\
\frac{\partial \mathrm{f}}{\partial X_{2}^{c}}(\mathbb{X})+\frac{\partial \mathrm{f}}{\partial X_{2}}(\mathbb{X}) \\
-1 \\
\frac{\partial \mathrm{f}}{\partial X_{1}^{1}}(\mathbb{X}) \\
\frac{\partial \mathrm{f}}{\partial X_{2}^{1}}(\mathbb{X}) \\
\vdots \\
\frac{\partial \mathrm{f}}{\partial X_{3}^{n}}(\mathbb{X})
\end{array}\right]
$$

We remark that the first three components of this vector take into account only the contributions of the slave node and the others components take into account the contributions of master nodes.

When a penalty method is used, the elementary residual contact vector is thus a $3(n+1)$ component vector given by:

$$
\mathbf{R}_{\boldsymbol{c}}^{\boldsymbol{d}}=-\varepsilon_{n} g_{n}^{d} \mathbf{N}_{\boldsymbol{c}}^{\boldsymbol{d}}
$$

where $\varepsilon_{n}$ is the penalty parameter. 


\subsection{Contact elementary tangent matrix}

The evaluation of the contact elementary tangent matrix requires the determination of the second variation of $g_{n}^{d}$.

\subsubsection{Second variation of $g_{n}^{d}$}

The derivation of relation (26) leads to

$$
\Delta \delta g_{n}^{d}=\Delta\left\{\left(\delta \boldsymbol{x}^{c}-\delta \boldsymbol{x}^{\boldsymbol{d}}\right) \cdot \boldsymbol{\nu}^{\boldsymbol{d}}\right\}
$$

which is equivalent to:

$$
\Delta \delta g_{n}^{d}=g_{n}^{d} \delta \boldsymbol{\nu}^{\boldsymbol{d}} \cdot \Delta \boldsymbol{\nu}^{\boldsymbol{d}}-\Delta \delta \boldsymbol{x}^{\boldsymbol{d}} \cdot \boldsymbol{\nu}^{\boldsymbol{d}} .
$$

If we introduce (29), $\Delta \delta \boldsymbol{x}^{d}$ can be evaluated:

$$
\begin{aligned}
\Delta \delta \boldsymbol{x}^{d}= & \Delta\left\{\delta \bar{X}_{1} \boldsymbol{\tau}_{1}^{d}+\delta \bar{X}_{2} \tau_{2}^{d}+\mathrm{d} \overline{\mathbf{f}}\right\} \\
= & \delta \bar{X}_{1} \Delta \boldsymbol{\tau}_{1}^{d}+\delta \bar{X}_{2} \Delta \boldsymbol{\tau}_{2}^{d} \\
& +\Delta \delta \bar{X}_{1} \tau_{1}^{d}+\Delta \delta \bar{X}_{2} \tau_{2}^{d}+\Delta \mathrm{d} \overline{\mathbf{f}}
\end{aligned}
$$

The problem is to determine $\Delta \mathrm{d} \overline{\mathbf{f}}$ and particularly the third component. The variation of the gradient can be calculated as

$$
\Delta \mathrm{d} \overline{\mathbf{f}}=\left[\begin{array}{c}
0 \\
0 \\
\delta \boldsymbol{x}^{t}\left\{\mathrm{H}_{\overline{\mathrm{f}}} \Delta \boldsymbol{x}+\mathbf{T}_{\mathbf{1}} \Delta \bar{X}_{1}+\mathbf{T}_{\mathbf{2}} \Delta \bar{X}_{2}\right\}
\end{array}\right]
$$

where

$$
\mathbf{T}_{\boldsymbol{l}}=\left[\begin{array}{c}
\frac{\partial^{2} \mathrm{f}}{\partial X_{l} \partial X_{1}^{c}}(\mathbb{X}) \\
\frac{\partial^{2} \mathrm{f}}{\partial X_{l} \partial X_{2}^{c}}(\mathbb{X}) \\
0 \\
\frac{\partial^{2} \mathrm{f}}{\partial X_{l} \partial X_{1}^{1}}(\mathbb{X}) \\
\frac{\partial^{2} \mathrm{f}}{\partial X_{l} \partial X_{2}^{1}}(\mathbb{X}) \\
\vdots \\
\frac{\partial^{2} \mathrm{f}}{\partial X_{l} \partial X_{3}^{n}}(\mathbb{X})
\end{array}\right] \quad \text { with } l=1,2
$$

$\mathrm{H}_{\overline{\mathrm{f}}}$ is the Hessian $(n+1), 3(n+1)$ matrix of $\mathrm{f}$.

$$
\mathrm{H}_{\overline{\mathrm{f}}}=\left[\begin{array}{ccc}
\frac{\partial^{2} \mathrm{f}}{\partial X_{1}^{c} \partial X_{1}^{c}}(\mathbb{X}) \cdots \frac{\partial^{2} \mathrm{f}}{\partial X_{k}^{i} \partial X_{1}^{c}}(\mathbb{X}) \cdots \frac{\partial^{2} \mathrm{f}}{\partial X_{3}^{n} \partial X_{1}^{c}}(\mathbb{X}) \\
\vdots & \vdots & \vdots \\
\frac{\partial^{2} \mathrm{f}}{\partial X_{1}^{c} \partial X_{3}^{n}}(\mathbb{X}) \cdots \frac{\partial^{2} \mathrm{f}}{\partial X_{k}^{i} \partial X_{3}^{n}}(\mathbb{X}) \cdots \frac{\partial^{2} \mathrm{f}}{\partial X_{3}^{n} \partial X_{3}^{n}}(\mathbb{X})
\end{array}\right]
$$

\subsubsection{Discrete expression and contact tangent matrix}

The variations of $\bar{X}_{1}, \bar{X}_{2}$ and vector $\nu^{d}$ can be expressed as follows:

$$
\begin{aligned}
\delta \bar{X}_{l}=\delta \boldsymbol{x}^{t} \mathbf{S}_{\boldsymbol{l}} \quad \text { et } \Delta \bar{X}_{l} & =\mathbf{S}_{\boldsymbol{l}}^{t} \Delta \boldsymbol{x} \quad l=1,2 \\
\delta \boldsymbol{\nu}^{\boldsymbol{d}}=\mathrm{U} \delta \boldsymbol{x} & \text { and } \Delta \boldsymbol{\nu}^{\boldsymbol{d}}=\mathrm{U} \Delta \boldsymbol{x}
\end{aligned}
$$

where $\mathbf{S}_{\boldsymbol{l}}(l=1,2)$ is a $3(n+1)$ component vector and $\mathrm{U}$ a matrix belonging to $\mathbb{M}_{3,3(n+1)}$.

Using equation (21), it is possible to obtain the variation of the tangent vectors:

$$
\Delta \tau_{l}^{d}=\left[\begin{array}{c}
0 \\
0 \\
\mathbf{D}_{\tau_{l}}{ }^{t} \Delta \boldsymbol{x}
\end{array}\right] \quad l=1,2 .
$$

In this relation, $\mathbf{D}_{\tau_{l}}$ is a $3(n+1)$ component vector depending $\mathbf{S}_{\boldsymbol{l}}$ and $3(n+1)$ and is given by:

$$
\begin{aligned}
& \mathbf{D}_{\boldsymbol{\tau}_{\mathbf{1}}}=\frac{\partial^{2} \mathrm{f}}{\partial X_{1} \partial X_{1}}(\mathbb{X}) \mathbf{S}_{\mathbf{1}}+\frac{\partial^{2} \mathrm{f}}{\partial X_{1} \partial X_{2}}(\mathbb{X}) \mathbf{S}_{\mathbf{2}}+\mathbf{T}_{\mathbf{1}} \\
& \mathbf{D}_{\boldsymbol{\tau}_{\mathbf{2}}}=\frac{\partial^{2} \mathrm{f}}{\partial X_{2} \partial X_{1}}(\mathbb{X}) \mathbf{S}_{\mathbf{1}}+\frac{\partial^{2} \mathrm{f}}{\partial X_{2} \partial X_{2}}(\mathbb{X}) \mathbf{S}_{\mathbf{2}}+\mathbf{T}_{\mathbf{2}}
\end{aligned}
$$

The relations introduced before leads to the following discrete expression of $\Delta \delta g_{n}^{d}$

$$
\Delta \delta g_{n}^{d}=\delta \boldsymbol{x}^{t} \mathrm{M}_{c}^{d} \Delta \boldsymbol{x}
$$

where $\mathbf{M}_{c}^{d}$ is a matrix $\mathbb{M}_{3(n+1), 3(n+1)}$.

$$
\begin{aligned}
\mathrm{M}_{c}^{d} & \frac{-1}{\| \boldsymbol{\tau}_{\mathbf{1}}^{d \wedge \boldsymbol{\tau}_{\mathbf{2}} \|}}\left(\mathrm{H}_{\overline{\mathrm{f}}}+\mathbf{T}_{\mathbf{1}} \mathbf{S}_{\mathbf{1}}{ }^{t}\right. \\
& \left.+\mathbf{T}_{\mathbf{2}} \mathbf{S}_{\mathbf{2}}{ }^{t}+\mathbf{S}_{\mathbf{1}} \mathbf{D}_{\boldsymbol{\tau}_{\mathbf{1}}}{ }^{t}+\mathbf{S}_{\mathbf{2}} \mathbf{D}_{\boldsymbol{\tau}_{\mathbf{2}}}{ }^{t}\right)+g_{n}^{d} \mathrm{U}^{t} \mathrm{U}
\end{aligned}
$$

Finally, when a penalty method is used, $\mathrm{K}_{c}^{d}$, the elementary diffuse contact tangent matrix belonging to $\mathbb{M}_{3(n+1), 3(n+1)}$ is:

$$
\mathrm{K}_{c}^{d}=\varepsilon_{n} g_{n}^{d} \mathrm{M}_{c}^{d}+\varepsilon_{n} \mathbf{N}_{c}^{d} \mathbf{N}_{c}^{d t} .
$$

\section{Numerical examples}

The approach presented here has been implemented in the finite element code SYSTUS developped by the company ESI Group and tested on both academic and industrial examples. We assume the contact to be frictionless.

\subsection{Contact between a plate and a rigid cylinder}

We consider the contact between a plate and a rigid cylinder. The rigid cylinder is choosen as the master body. The plate is submitted to an imposed pressure on a part, $S_{p}$, of its boundary and is clamped on another part (See Fig. 11). The pressure is applied gradually until $P=200 \mathrm{MPa}$ by steps of $20 \mathrm{MPa}$. The mechanical properties of the plate are: $E=200000 \mathrm{MPa}$ and $\nu=0.3$. 


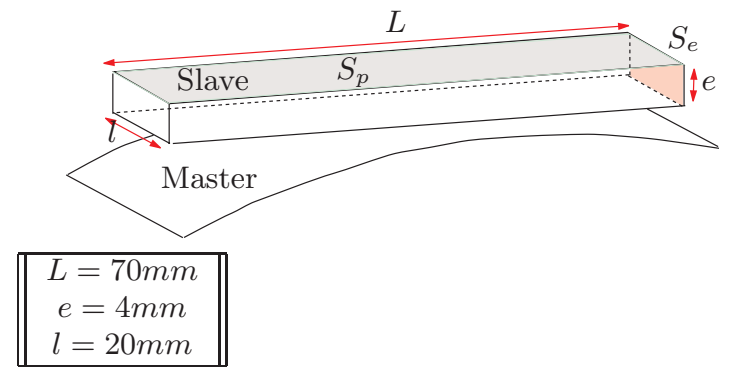

Fig. 11. Contact between a plate and a rigid cylinder: geometry.

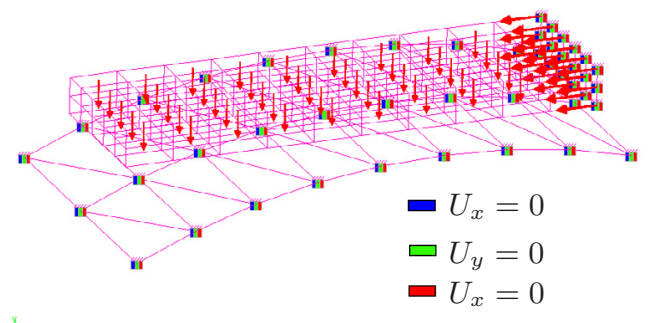

\begin{tabular}{||c|c|}
\hline Number of elements & 132 \\
Number of nodes & 225 \\
\hline
\end{tabular}

Fig. 12. Contact between a plate and a rigid cylinder: finite element model.

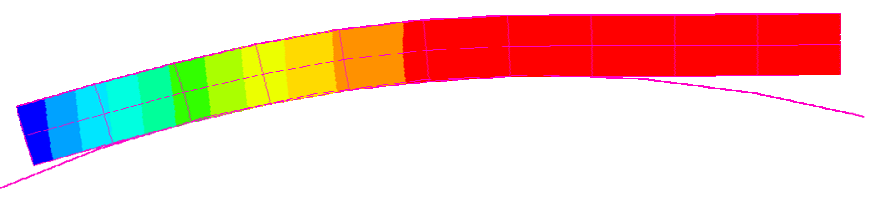

Fig. 13. Contact between a plate and a rigid cylinder: deformed configuration.

The finite element model can be seen in Figure 12: the master surface is composed of 32 triangular facets and the slave body of 100 quadrangle facets. There are 225 nodes.

The shape of the master surface can generate diffculties. To point out these problems, only a part of the master surface is studied (Fig. 13). A zoom on the deformed configuration obtained with a classic contact algorithm

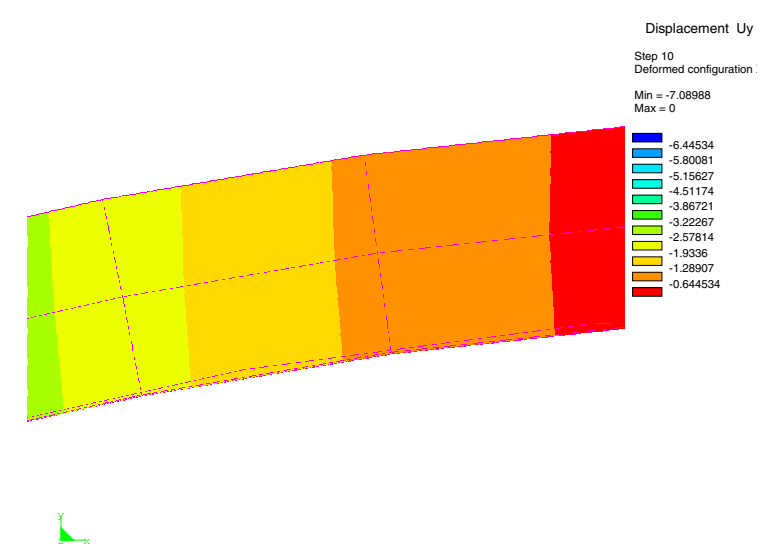

(a) Classic contact element

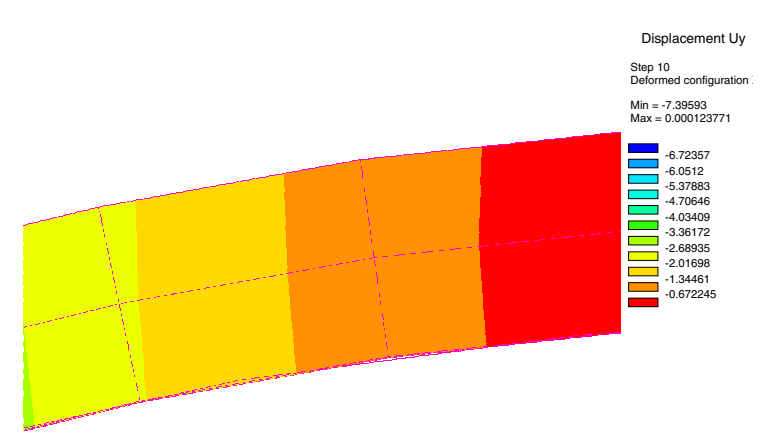

(b) 3D diffuse contact element

Fig. 14. Zoom in deformed configuration.

is represented in Figure 14(a). It appears clearly that the non smoothness of the cylinder induces an important interpenetration. This problem is strongly attenuated when the new contact element is used. We can see in Figure 14(b) that the contact does not occur on a flat facet, but on a surface which fits the shape of the cylinder. This problem is strongly attenuated by using the new contact element. It can be seen especially in figure. The contact does not take place on a fat facet, but on a surface which completely follows the shape of the cylinder

\subsection{Pinch of pipes}

This example deals with contact problem between two deformable bodies in 3D. A pipe is pinched between two identical parallel plates (Fig. 15). Only one quarter of the model is used because of symmetry reason (Fig. 16). The plates and the pipe have the same elastoplastic properties:

- Young Modulus: $E=200000 \mathrm{MPa}$. 


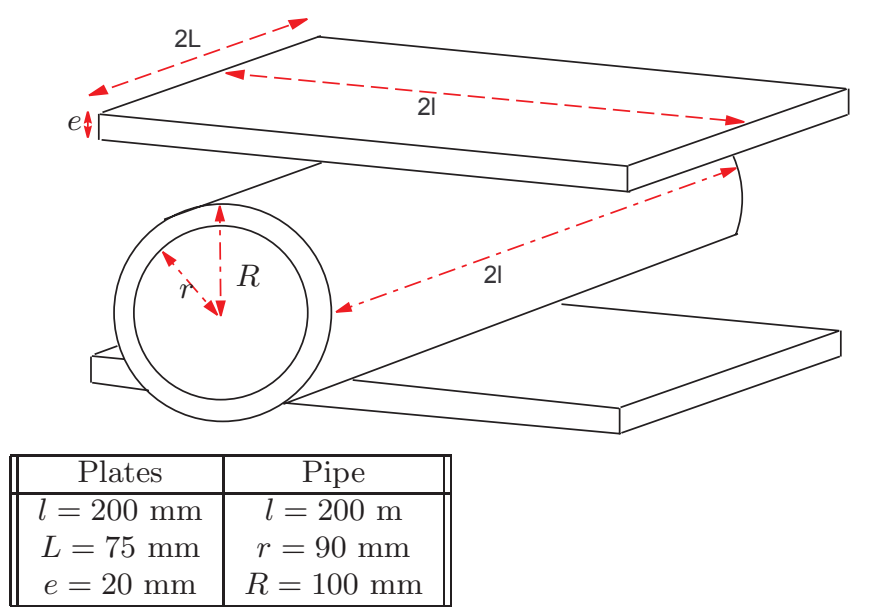

Fig. 15. Pinch of pipes: geometrical model.

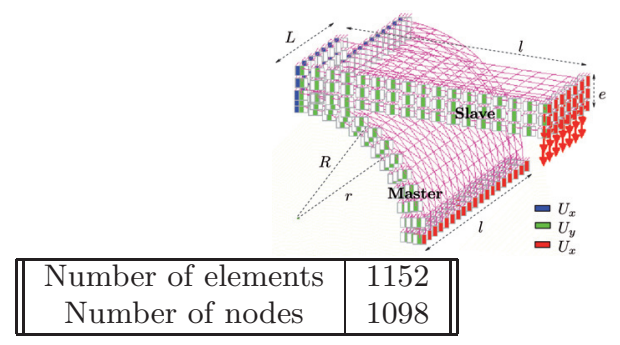

Fig. 16. Pinch of pipes: finite element model.

- Poisson ration: $\nu=0.30$.

- Yield stress: $\sigma_{y}=400 \mathrm{MPa}$.

- Slope hardening: $H=3450 \mathrm{MPa}$.

The load consists in a vertical imposed displacement of $100 \mathrm{~mm}$ at the end of the plate (Fig. 16). In the elastic study, the load step is equal to $2.5 \mathrm{~mm}$ and reduced to $1 \mathrm{~mm}$ when dealing with elastoplasticity. This test has been performed with a traditional contact algorithm and with our approach. The computations have been performed for both cases.

We observe that the number of iterations is reduced of $14 \%$ when the surface smoothing procedure is used. The CPU time is not increased. The deformed configuration can be seen in Figure 17.

\section{Conclusion}

In this work, a 3D smoothing contact surface procedure based on diffused approximation associated with an original contact search algorithm by convex hulls has been presented. Our approach proved to overcome the numerical instabilities and lacks of precision encountered when dealing with contact problems. We also experienced that the robustness and the efficiency of the contact search are considerably improved. Problems due to strong variations of vector fields are reduced. Nodes may slide smoothly on the diffuse surface without impeding the convergence of the global process.

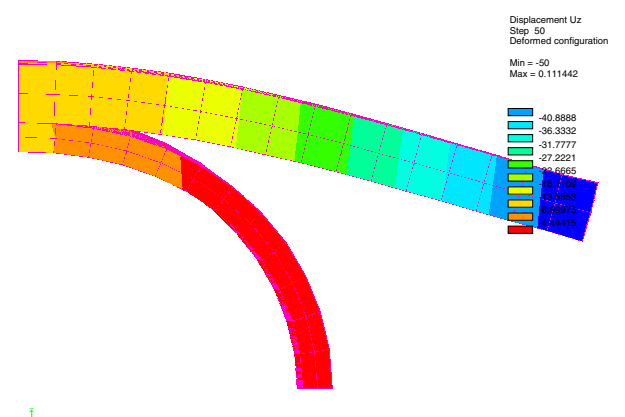

(a) $U_{z}=60 \mathrm{~mm}$

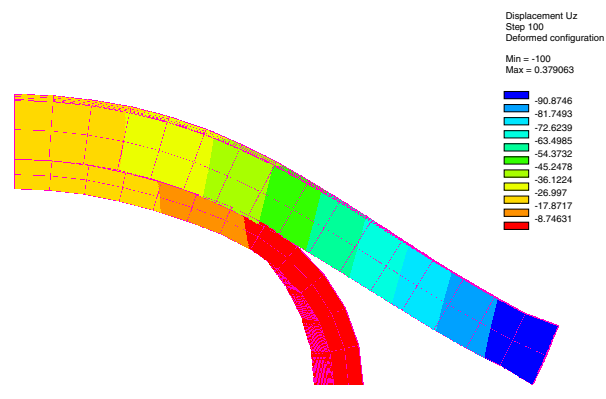

(b) $U_{z}=100 \mathrm{~mm}$

Fig. 17. Pinch of pipes: deformed configurations.

\section{References}

1. P. Alart, A. Curnier, A mixed formulation for frictional contact problems prone to Newton like solution methods. Comput. Meth. Appl. Mech. Eng. 92, 353-375 (1991).

2. T. Belytschko, W.J.T. Daniel, G. Ventura, A monolithic smoothing-gap algorithm for contact-impact based on the signed distance function. Int. J. Numer. Meth. Eng. 55, 101-125 (2002).

3. T. Belytschko, M.O. Neal, Contact-impact by the piball algorithm with penalty and lagrangian methods. Int. J. Numer. Meth. Eng. 31, 547-572 (1991).

4. D.J. Benson, J.O. Hallquist, A single surface contact algorithm for the post-buckling analysis of shell structures. Comput. Meth. Appl. Mech. Eng. 78, 141-163 (1990).

5. P. Breitkopf, A. Rassineux, J.M. Savignat, P. Villon, Integration and convergence constraints in Diffuse Element Method. Comput. Meth. Appl. Mech. Eng. 193, 1203-1220 (2004).

6. P. Breitkopf, A. Huerta, Meshfree and Particle Based Approaches in Computational Mechanics. Kogan Page Science, ISBN 1903996457 (2004).

7. D. Chamoret, A. Rassineux, J.M. Bergheau, P. Villon, Modelling of contact surface by local hermite diffuse interpolation. Proceedings of ESAFORM 2001, The 
$4^{\text {th }}$ International ESAFORM Conference on Material Forming, University of Liège, Belgium 1, 179-182 (2001).

8. D. Chamoret, P. Saillard, A. Rassineux, J.M. Bergheau, New smoothing procedures in contact mechanics. J. Comput. Appl. Math. 168, 107-116 (2004).

9. C. Chappuis, A. Rassineux, P. Breitkopf, P. Villon, Improving surface remeshing by feature recognition. Eng. Comput. 20, 202-209 (2004).

10. A. Curnier, Q.C. He, A. Klarbring, Continuum mechanics modelling of large deformation contact with friction, in Contact mechanics, M. Raous, M. Jean and J.J Moreau, Eds. Plenum Press (1995), pp. 145-158.

11. R. Diekmann, J. Hungershö, M. Lux, L. Taenzer, J.M. Wierum, Efficient contact searching for finite element Analysis. European Congress on Computational Methods in Applied Sciences and Engineering, Barcelona, Spain (2000).

12. N. El-Abbasi, S.A. Meguid, A. Czekanski, On the modelling of smooth contact surfaces using cubic splines. Int. J. Numer. Meth. Eng. 50, 953-967 (2001).

13. E.G. Nezami, Y.M.A. Hashash, D. Zhao, J. Ghaboussi, A fast contact detection algorithm for 3-D discrete element method. Comput. Geotech. 31, 575-587 (2004).

14. L. Fourment, J.L. Chenot, K. Mocellin, Numerical formulations and algorithms for solving contact problems in metal forming simulation. Int. J. Numer. Meth. Eng. 46, 1435-1462 (1999).

15. A. Heege, P. Alart, A Frictional contact element for strongly curved contact problems. Int. J. Numer. Meth. Eng. 39, 165-184 (1996).

16. A. Klarbring, Large displacement frictional contact: a continuum framework for finite element discretization. Eur. J. Mech. A 14, 237-253 (1995).

17. G. Kloosterman, A.H. Van Den Boogaard, J. Huetink, An efficient contact search algorithm. The $5^{\text {th }}$ international ESAFORM conference on MATERIAL FORMING. M. Pietrzyk, Z. Mitura, J. Kaczmar, Cracow, Poland (2002), pp. 99-102.

18. L. Krstulovic-Opara, P. Wriggers, J. Korelc, A $C^{1}$ continuous formulation for $3 D$ finite deformation frictional contact. Comput. Mech. 29, 27-42 (2002).

19. T.A. Laursen, Computational Contact and Impact Mechanics. Springer Verlag (2002).

20. T.A. Laursen, J.C. Simo, A Continuum-based finite element formulation for the implicit solution of multibody, large deformation frictional contact problems. Int. J. Numer. Meth. Eng. 36, 3451-3485 (1993).
21. Li S, Dong Qian, Wing Kam Liu, Belytschko T. A meshfree contact-detection algorithm. Comput. Meth. Appl. Mech. Eng. 190, 3271-3292.

22. W.N. Liu, G. Metschke, H.A. Mang, A note on the algorithmic stabilization of $2 \mathrm{D}$ contact analyses, in Computational Methods in Contact Mechanics, L. Gaul, C.A. Brebbia, Wessex Institute of Technology, WITpresss (1999), pp. 231-240.

23. B. Nayrolles, G. Touzot, P. Villon, Generalizing the finite element method: diffuse approximation and diffuse elements. J. Comput. Mech. 10, 307-138 (1992).

24. M. Oldenburg, L. Nilsson, The position code algorithm for contact searching. Int. J. Numer. Meth. Eng. 37, 359-386 (1994).

25. V. Padmanabhan, T.A. Laursen, A framework for development of surface smoothing procedures in large deformation frictional contact analysis. Finite Elem. Anal. Design 37, 173-198 (2001).

26. P. Papadopoulos, R.L. Taylor, A mixed formulation for the finite element solution of contact problems. Comput. Meth. Appl. Mech. Eng. 94, 373-389 (1992).

27. G. Pietrzak, A. Curnier, Large deformation frictional contact mechanics: continuum formulation and augmented Lagrangian treatment. Comput. Meth. Appl. Mech. Eng. 177, 351-381 (1999).

28. M.A. Puso, T.A. Laursen, A 3D contact smoothing method using Gregory patches. Int. J. Numer. Meth. Eng. 54, 1161-1194 (2002).

29. A. Rassineux, P. Villon, J.M. Savignat, O. Stab, Surface remeshing by local hermite diffuse interpolation. Int. J. Numer. Meth. Eng. 49, 31-49 (2000).

30. Sheng Ping Wang, Eiiji Nakamachi. The inside-outside contact search algorithm for finite element analysis. Int. J. Numer. Meth. Eng. 40, 3665-3685 (1997).

31. P. Wriggers, Computat. Contact Mechanics. Wiley (2002).

32. P. Wriggers, L. Krstulovic-Opara, J. Korelc, Smooth $C^{1}$ interpolations for two-dimensional frictional contact problems. Int. J. Numer. Meth. Eng. 51, 1469-1495 (2001).

33. P. Wriggers, T. Vu Van, E. Stein, Finite element formulation of large deformation impact-contact problems with friction. Comput. Struct. 37, 319-331 (1990).

34. Z.H. Zhong, L. Nilsson, A contact searching algorithm for general 3-D contact-impact problems. Comput. Struct. 34, 327-335 (1990).

35. Z.H. Zhong, L. Nilsson, Automatic contact searching algorithm for dynamic finite element analysis. Comput. Struct. 52, 187-197 (1994). 